\title{
Phase retrieval algorithms for direct phasing of coherent nanocrystal diffraction
}

\author{
J. P. J. Chen ${ }^{1}$ and R. A. Kirian ${ }^{1}$ \\ ${ }^{1}$ Department of Physics, Arizona State University, Tempe, Arizona, USA
}

As first noted by David Sayre in 1952 [1], diffracted intensities between Bragg reflections can be employed to directly solve the phase problem in crystallography. These so-called inter-Bragg intensities, in conjunction with the Bragg reflections, uniquely determine the continuous molecular transform of the unit cell and existing iterative phase retrieval algorithms developed for single particle imaging [2] can then be used to recover the unit cell's electron density [3-6]. Diffracted intensities between Bragg reflections have now been observed from nanocrystals of biomolecules illuminated by the intense and coherent radiation from x-ray free-electron lasers (XFELs) [7]. However, a pressing hindrance of this phasing method is that intensities between Bragg peaks depend crucially on the spatial arrangement of every molecule in the crystal, including ones on the edge of the crystal where they may not form a complete unit cell $[4,8,9]$. Therefore, due to the nature of the data collection process from XFELs where a reciprocal space intensity volume is assembled from an ensemble of crystals of different sizes and shapes, new algorithms need to be considered in order to extract the information contained within the inter-Bragg reflections. The fundamentals of this puzzle will be introduced and current algorithmic developments in dealing with it outlined in this presentation.

\section{Acknowledgment}

RAK acknowledges support from NSF STC Award (1231306).

\section{References}

[1] Sayre D., (1952). Acta Cryst., 5, 843.

[2] Marchesini S., (2007). Rev. Sci. Instrum., 78, 011301.

[3] Spence J.C.H., et al., (2011). Opt. Express, 19, 2866-2873.

[4] Kirian R.A., et al., (2014). Phil. Trans. R. Soc. B, 369, 20130331.

[5] Chen J.P.J., et al., (2014). Acta Cryst., A70, 154-161.

[6] Kirian R.A., et al., (2015). Phys. Rev. X, 5, 011015.

[7] Chapman H.N., et al., (2011). Nature, 470, 73-78.

[8] Elser V., (2013). Acta Cryst., A69, 559-569.

[9] Chen J.P.J. and Millane R.P., (2014). J. Opt. Soc. Am. A, 31, 1730-1737. 